\title{
ABSOLUTE CONTINUITY OF QUASICONFORMAL MAPPINGS ON CURVES
}

\author{
Zoltán M. Balogh, Pekka Koskela and Sari Rogovin
}

\begin{abstract}
We show that a quasiconformal mapping between two proper, locally Ahlfors $Q$-regular metric spaces, $Q>1$, is absolutely continuous on almost every curve. We further relax the limes superior in the definition of quasiconformality to a limes inferior and verify that exceptional sets analogous to the Euclidean setting can be allowed.
\end{abstract}

\section{Introduction}

The history of various definitions of quasiconformality is long. Grötzsch and Teichmüller considered smooth mappings in the 1920's and 1930's and used an analytic definition. In 1954, Ahlfors [A] initiated the study of nonsmooth quasiconformal mappings in the plane using a geometric definition. We take as our starting point the following metric definition.

A homeomorphism $f: X \rightarrow X Y$ between metric spaces $\left(X, d_{X}\right)$ and $\left(Y, d_{Y}\right)$ is said to be quasiconformal if it satisfies

$$
H_{f}(x):=\limsup _{r \rightarrow 0} H_{f}(x, r) \leq H<\infty
$$

for all $x \in X$ for some $H$ independent of $x$, where

and

$$
\begin{gathered}
H_{f}(x, r)=\frac{L_{f}(x, r)}{l_{f}(x, r)}, \\
L_{f}(x, r)=\sup \left\{d_{Y}(f(x), f(y)): d_{X}(x, y) \leq r\right\}
\end{gathered}
$$

$$
l_{f}(x, r)=\inf \left\{d_{Y}(f(x), f(y)): d_{X}(x, y) \geq r\right\} .
$$

Keywords and phrases: Absolute continuity, quasiconformal mappings, metricmeasure spaces

AMS Mathematics Subject Classification: 30C65, 26B30

The authors were supported by grants from the Swiss NSF and the Academy of Finland. Part of the research was done while S.R. was visiting at the University of Bern. 
The equivalence of the metric (and geometric) definition in $\mathbb{R}^{n}, n \geq 2$, with the analytic definition which requires that $f \in W_{l o c}^{1, n}\left(\mathbb{R}^{n} ; \mathbb{R}^{n}\right)$ and that $|D f(x)|^{n} \leq K J_{f}(x)$ almost everywhere, was established by Gehring in the 1960s [G1,2]. Especially, Gehring proved that a quasiconformal mapping is absolutely continuous on almost all lines by a technique that goes back to Menchoff [Me]. This method relies on the foliation of $\mathbb{R}^{n}$ by parallel lines.

The first to consider quasiconformal mappings in a non-Riemannian setting was Mostow $[\mathrm{Mo1}, 2]$ in connection to rigidity theorems. In a similar way, Bourdon and Pajot [BouP] used quasiconformal mappings to prove a rigidity result for hyperbolic buildings. Various definitions of quasiconformality on the Heisenberg and Carnot groups were also used by Pansu $[\mathrm{P}]$, Korányi and Reimann [KoR] and Vodopyanov and Greshnov [VG]. Regarding the metric definition, the associated foliation of the space is more complicated in the group setting and the approach used by Gehring faces formidable difficulties. Nevertheless, Mostow [Mo3] and Margulis and Mostow $[\mathrm{MM}]$ were able to prove an analog of the absolute continuity on almost all lines by a variant of this technique. It then followed that a version of the analytic definition was also satisfied. An alternate approach was given by Heinonen and Koskela [HeK1]. This was based on first proving that, for a Carnot group $G=X=Y$, quasiconformality guarantees the global condition of quasisymmetry, i.e. that $H_{f}(x, r) \leq H^{\prime}<\infty$ for all $x, r$, which allowed one to invoke the regularity results by Pansu $[\mathrm{P}]$. This method was shown in [HeK2] to be robust enough to extend to a class of Ahlfors $Q$-regular metric spaces that support a suitable Poincaré inequality. In fact, (localizing) this general setting covers all the above absolute continuity results.

A problem of general interest is then to give minimal assumptions on metric spaces $X$ and $Y$ and on a homeomorphism $f$ between these spaces so as to guarantee absolute continuity on almost all curves or even quasisymmetry. The goal would be to relax the Poincaré inequality or the foliation properties of $X$ by geodesics as well as to relax (1).

In the Euclidean setting, it was shown by Heinonen and Koskela in 1995 that, surprisingly, $H_{f}(x)$ can be replaced with

$$
h_{f}(x):=\liminf _{r \rightarrow 0} H_{f}(x, r)
$$

in the definition of quasiconformality, see [HeK1]. It should come as no surprise that the resulting new definition is easier to verify in practical situations and of importance in complex dynamics $[\mathrm{H}],[\mathrm{GrS}],[\mathrm{PrR}]$. This improvement was obtained by invoking the powerful Besicovitch covering 
theorem that is in nature Euclidean and already fails in Heisenberg groups $[\mathrm{KoR}],[\mathrm{R}]$. The question whether this improvement could also hold in nonEuclidean settings was then raised. This has remained an open problem until now. The technique from [HeK1] was however shown by Balogh and Koskela $[\mathrm{BK}]$ to yield an intermediate result where the limit superior can be replaced with a less stringent limit. Let us mention at this point that the phenomenon of replacing the "limsup" by a "liminf" condition seems to be typical for the class of quasiconformal maps. Balogh and Csörnyei showed recently (see $[\mathrm{BC}]$ ) that this is no longer possible for Lipschitz functions or in Sobolev classes.

Furthermore, in the Euclidean setting, one can allow for an exceptional set in the definition. Already Gehring [G1,2] showed that the uniform boundedness of $H_{f}(x)$ in the definition of quasiconformality can be relaxed to the assumption that $H_{f}(x)<\infty$ outside a set $E$ of $\sigma$-finite $(n-1)$ dimensional measure and that $H_{f}(x) \leq H$ almost everywhere. Kallunki and Koskela have recently showed that this also works for $h_{f}[\mathrm{KK} 1,2]$.

Our first result gives a striking generalization of the above results.

Theorem 1.1. Let $X, Y$ be locally Ahlfors $Q$-regular metric spaces, $Q>$ 1. Suppose that $X$ is proper and a homeomorphism $f: X \rightarrow Y$ satisfies $h_{f}(x)<\infty$ for all $x \in X \backslash E$, where $E$ has $\sigma$-finite $(Q-1)$-dimensional Hausdorff measure and that $h_{f}(x) \leq H<\infty$ almost everywhere. Then $f \in W_{l o c}^{1,1}(X ; Y)$.

Because each homeomorphism in the Sobolev class $W_{l o c}^{1,1}(X ; Y)$ is absolutely continuous on 1-modulus almost every curve, Theorem 1.1 encompasses all previous results. For the definition of $W_{l o c}^{1,1}(X ; Y)$, see section 3 below.

The local Ahlfors $Q$-regularity of a space $X$ requires that $X$ be equipped with a Borel regular measure with the property that, given a compact set $A$, there are constants $C>0$ and $\delta>0$ so that

$$
C^{-1} r^{Q} \leq \mu(B(x, r)) \leq C r^{Q}
$$

whenever $x \in A$ and $0<r<\delta$. The properness requires that each closed ball in $X$ be compact.

As pointed out above, no result in terms of $h_{f}$ was known outside the Euclidean space. Moreover, the earlier results did not allow for an exceptional set even for $H_{f}$.

Our proof of Theorem 1.1 is substantially different from the two approaches described above. Gehring's method, together with its variants, require the space $X$ to have a nice foliation by curves. We assume no such 
structure for $X$. On the other hand, the technique used in $[\mathrm{HeK} 1,2]$ relies heavily on the Poincaré inequality that now gets entirely disposed of, as we assume only the Ahlfors regularity of $X$ and $Y$. The $h_{f}$-result further bases on the Besicovitch covering theorem that surely fails in our generality. Let us mention here two key points in our proof. We prove a new covering theorem that is tailored for our needs and essentially consider all the curves in $X$ at once. As a consequence, we obtain a new proof even in the Euclidean setting that is simpler than the argument in $[\mathrm{KK} 1,2]$.

In the case of Carnot groups, we obtain a much stronger result than Theorem 1.1.

Theorem 1.2. Let $f$ be a self-homeomorphism of a Carnot group $G$ with homogeneous dimension $Q>1$ such that $h_{f}(x)<\infty$ for all $x \in G \backslash E$, where $E$ has $\sigma$-finite $(Q-1)$-dimensional Hausdorff measure and that $h_{f}(x) \leq$ $H<\infty$ almost everywhere. Then $f$ is quasisymmetric, $f \in W_{l o c}^{1, Q}(G ; G)$, and $\left|f_{*}(x)\right|^{Q} \leq K J_{f}(x)$ almost everywhere.

Here $f_{*}$ is the horizontal differential of $f$ at $x$ and $J_{f}$ is the determinant of $f_{*}$.

Theorem 1.1 and Theorem 1.2 are new even for Heisenberg groups [KoR], [HeK1], [MM], [BK], even if we replace $h_{f}$ with $H_{f}$. It seems that such a result cannot be established by a variant of the technique used by Gehring, Margulis and Mostow. To comment on the size of the exceptional set in the above statement, recall that the topological dimension of the Heisenberg group $\mathbb{H}^{n}, n \geq 1$, is $2 n+1$ and the homogeneous is $2 n+2$. The best conclusion one could hope for, from a Gehring type argument, would then be an exceptional set of Euclidean dimension $2 n=Q-2$. Notice, however, that there are examples of sets in $\mathbb{H}^{n}$ of Heisenberg dimension $Q-1$ whose Euclidean dimension is strictly larger than $Q-2$, see [BRS], [BT], [KiS].

Theorem 1.2 will be obtained as a corollary to our more general results. Indeed, the statement continues to hold when $G$ is replaced with a proper, $Q$-regular metric space that supports a 1-Poincaré inequality. Regarding the regularity $f \in W_{l o c}^{1, Q}$, one only needs to assume, in addition to the 1-Poincaré inequality for the initial space, that the target space be $Q$ regular. This appears to be a new conclusion even in the Euclidean setting. Our approach also allows for a version of Theorem 1.2 under a $p$-Poincaré inequality assumption for $1<p \leq Q$.

The paper is organized as follows. In section 2 we introduce a new covering lemma and its consequence for quasiconformal mappings. This will 
allow us to bypass the lack of the Besicovitch covering theorem. We discuss path families in section 3 and prove a proposition that will be crucial for allowing an exceptional set. Theorem 1.1 is proven in section 4. Finally, in section 5, we prove a quasisymmetry result, and deduce Theorem 1.2 from it.

Acknowledgment. The authors wish to thank Juha Heinonen, Steve Buckley and Matthieu Rickly for reading an earlier version of the manuscript and for valuable comments.

\section{Covering Lemmas}

Let us begin by recalling the usual Vitali covering theorem, see [Ma, p. 2325]. In what follows, $A \subset \subset X$ requires that the closure of $A$ be compactly contained in $X$, and, given a ball $B=B(x, r)$ and $\lambda>0$, we use the notation $\lambda B$ for $B(x, \lambda r)$.

Lemma 2.1. Let $\mathcal{B}$ be a family of closed balls in a metric space $X$ so that $\cup_{B \in \mathcal{B}} B \subset \subset X$. Then there is a finite or countable sequence $B_{i} \in \mathcal{B}$ of pairwise disjoint balls such that

$$
\cup_{B \in \mathcal{B}} B \subset \cup_{i} 5 B_{i} .
$$

The following variant of the above Vitali covering theorem will be crucial for us. The point here is that we can ask for more information on the selected sequence when we confine ourselves with only covering the set of centers of the original balls.

Lemma 2.2. Let $\mathcal{B}$ be a collection of balls $B\left(x, r_{x}\right)$ (open or closed) with $x \in A$ in a metric space $X$ such that

$$
\cup_{B \in \mathcal{B}} B \subset \subset X \text {. }
$$

Then there exists a finite or countable sequence $B_{i}=B\left(x_{i}, r_{i}\right) \in \mathcal{B}$ with the following properties:

(i) $A \subset \cup_{i} B\left(x_{i}, r_{i}\right)$;

(ii) If $i \neq j, i, j \in \mathbb{N}$, it follows that

(a) $x_{i} \in X \backslash B\left(x_{j}, r_{j}\right)$ and $B\left(x_{j}, r_{j}\right) \backslash B\left(x_{i}, r_{i}\right) \neq \emptyset$

or (b) $x_{j} \in X \backslash B\left(x_{i}, r_{i}\right)$ and $B\left(x_{i}, r_{i}\right) \backslash B\left(x_{j}, r_{j}\right) \neq \emptyset$;

(iii) $B\left(x_{i}, \frac{1}{3} r_{i}\right) \cap B\left(x_{j}, \frac{1}{3} r_{j}\right)=\emptyset$ when $i \neq j$.

Proof. Denote $\mathcal{B}=\left\{B\left(x, r_{x}\right): x \in A\right\}$. Let $M=\sup _{x \in A} r_{x}<\infty$. Set

$$
A_{1}=\left\{x \in A: \frac{3}{4} M<r_{x} \leq M\right\} \text {. }
$$


Choose $x_{1} \in A_{1}, x_{2} \in A_{1} \backslash B\left(x_{1}, r_{x_{1}}\right), x_{3} \in A_{1} \backslash \cup_{i=1}^{2} B\left(x_{i}, r_{x_{i}}\right)$ and so on. For some $J \in \mathbb{N}$

$$
A_{1} \backslash \bigcup_{j=1}^{J} B\left(x_{j}, r_{x_{j}}\right)=\emptyset
$$

since $\cup_{B \in \mathcal{B}} B \subset \subset X, r_{x} \approx M$ for $x \in A_{1}$ and the balls $B\left(x_{j}, \frac{1}{3} r_{x_{j}}\right)$ are pairwise disjoint. However, our covering construction is not good enough, because (ii) is not necessarily satisfied. For this purpose, set $\mathcal{F}_{1}=\{1\}$ and

$$
\mathcal{F}_{j+1}=\left\{i \in \mathcal{F}_{j}: B\left(x_{i}, r_{x_{i}}\right) \not \subset B\left(x_{j+1}, r_{x_{j+1}}\right)\right\} \cup\{j+1\} .
$$

Now $\cup_{j=1}^{J} B\left(x_{j}, r_{x_{j}}\right)=\cup_{j \in \mathcal{F}_{J}} B\left(x_{j}, r_{x_{j}}\right)$ since we only remove those balls which are already covered. Now set $\mathcal{B}_{1}=\left\{B\left(x_{i}, r_{x_{i}}\right): i \in \mathcal{F}_{J}\right\}$. This family has property (ii). Property (iii) clearly also holds.

We continue inductively. Once $\mathcal{B}_{k}$ is chosen, repeat the above construction for

$$
A_{k+1}=\left\{x \in A \backslash\left(\cup_{i=1}^{k} \cup_{B \in \mathcal{B}_{i}} B\right):\left(\frac{3}{4}\right)^{k+1} M<r_{x} \leq\left(\frac{3}{4}\right)^{k} M\right\}
$$

to obtain $\mathcal{B}_{k+1}$.

Set $\mathcal{B}_{S}=\cup_{j=1}^{\infty} \mathcal{B}_{j}=\left\{B_{k}=B\left(x_{k}, r_{k}\right): k \in \mathbb{N}\right\}$. Clearly by construction $A \subseteq \cup_{B \in \mathcal{B}_{S}} B$. So let us check property (ii): Let $j \neq i$. If $B_{j}, B_{i} \in \mathcal{B}_{k}$ for some $k$ then (ii) is automatically valid by construction. If $B_{j} \in \mathcal{B}_{k}$ and $B_{i} \in \mathcal{B}_{l}$, we can assume that $k<l$, then by construction $x_{i} \notin B_{j}$ and

$$
d\left(x_{i}, x_{j}\right) \geq r_{j}>\left(\frac{3}{4}\right)^{k} M \geq\left(\frac{3}{4}\right)^{k-l+1} r_{i} \geq r_{i} .
$$

Thus $x_{j} \notin B_{i}$ and we conclude that (ii) is valid.

To prove (iii), let $B\left(x_{i}, r_{i}\right), B\left(x_{j}, r_{j}\right) \in \mathcal{B}_{S}$. By symmetry we may assume that they satisfy part (a) of (ii). Notice that (a) yields that $d\left(x_{i}, x_{j}\right) \geq r_{j}$ and $r_{i}<d\left(x_{i}, x_{j}\right)+r_{j}$. If there were a point $y \in B\left(x_{i}, \frac{1}{3} r_{i}\right) \cap B\left(x_{j}, \frac{1}{3} r_{j}\right)$, then we would conclude that

$$
d\left(x_{i}, x_{j}\right) \leq \frac{1}{3} r_{i}+\frac{1}{3} r_{j}<\frac{1}{3} d\left(x_{i}, x_{j}\right)+\frac{2}{3} r_{j} .
$$

Then $d\left(x_{i}, x_{j}\right)<r_{j}$, which is a contradiction.

The power of Lemma 2.2 is demonstrated by the following result.

Lemma 2.3. Let $f: X \rightarrow Y$ be a homeomorphism between metric spaces $X$ and $Y$ and $\mathcal{B}=\left\{B\left(x_{i}, r_{i}\right): i \in \mathbb{N}\right\}$ a family of (open or closed) balls. Assume that there exists $H \geq 1$ such that for each $B_{i}=B\left(x_{i}, r_{i}\right) \in \mathcal{B}$ we have that

$$
B\left(f\left(x_{i}\right), \frac{1}{2 H} \operatorname{diam}\left(f\left(B_{i}\right)\right)\right) \subset f\left(B_{i}\right)
$$

and $\mathcal{B}$ satisfies condition (ii) of Lemma 2.2. Then for $i \neq j$ we have

$$
B\left(f\left(x_{i}\right), \frac{\operatorname{diam}\left(f\left(B_{i}\right)\right)}{10 H^{2}}\right) \cap B\left(f\left(x_{j}\right), \frac{\operatorname{diam}\left(f\left(B_{j}\right)\right)}{10 H^{2}}\right)=\emptyset .
$$


Proof. Denote by $B_{j}=B\left(x_{j}, r_{j}\right)$ and $B_{i}=B\left(x_{i}, r_{i}\right)$. Assume that $x_{i} \notin B_{j}$ and let $z \in B_{j} \backslash B_{i}$. Then $f\left(x_{i}\right) \notin f\left(B_{j}\right)$ and $f(z) \in f\left(B_{j}\right) \backslash f\left(B_{i}\right)$. We have two cases to consider.

Case 1: $d_{Y}\left(f\left(x_{i}\right), f\left(x_{j}\right)\right)>\operatorname{diam} f\left(B_{i}\right) / 2 H$.

Since $x_{i} \notin B_{j}$ we have $d_{Y}\left(f\left(x_{i}\right), f\left(x_{j}\right)\right)>\operatorname{diam} f\left(B_{j}\right) / 2 H$. This implies by the triangle inequality that

$$
B\left(f\left(x_{i}\right), \frac{\operatorname{diam} f\left(B_{i}\right)}{5 H}\right) \cap B\left(f\left(x_{j}\right), \frac{\operatorname{diam} f\left(B_{j}\right)}{5 H}\right)=\emptyset .
$$

Case 2: $d_{Y}\left(f\left(x_{i}\right), f\left(x_{j}\right)\right) \leq \operatorname{diam} f\left(B_{i}\right) / 2 H$.

Since $f(z) \notin B\left(f\left(x_{i}\right), \operatorname{diam} f\left(B_{i}\right) / H\right)$ we see that

$$
d_{Y}\left(f(z), f\left(x_{j}\right)\right)+d_{Y}\left(f\left(x_{i}\right), f\left(x_{j}\right)\right) \geq \frac{\operatorname{diam} f\left(B_{i}\right)}{H},
$$

which implies

and therefore

$$
\operatorname{diam} f\left(B_{j}\right)+\frac{\operatorname{diam} f\left(B_{i}\right)}{2 H} \geq \frac{\operatorname{diam} f\left(B_{i}\right)}{H},
$$

From the above estimate we infer that

$$
\operatorname{diam} f\left(B_{j}\right) \geq \frac{\operatorname{diam} f\left(B_{i}\right)}{2 H} .
$$

$$
d_{Y}\left(f\left(x_{i}\right), f\left(x_{j}\right)\right) \geq \frac{\operatorname{diam} f\left(B_{j}\right)}{H} \geq \frac{\operatorname{diam} f\left(B_{i}\right)}{2 H^{2}} .
$$

And by the triangle inequality we obtain

finishing the proof.

$$
B\left(f\left(x_{i}\right), \frac{\operatorname{diam} f\left(B_{i}\right)}{10 H^{2}}\right) \cap B\left(f\left(x_{j}\right), \frac{\operatorname{diam} f\left(B_{j}\right)}{10 H^{2}}\right)=\emptyset,
$$

\section{Path Families and Sobolev Spaces}

Let $\Gamma$ be a path family in a metric measure space $(X, d, \mu)$. Here path refers to a continuous, non-constant map $\gamma: I \rightarrow X$, where $I \subset \mathbb{R}$ is a nondegenerate interval. We call a Borel function $\rho: X \rightarrow[0, \infty]$ admissible for the path family $\Gamma$, if

$$
\int_{\gamma} \rho d s \geq 1
$$

for each locally rectifiable $\gamma \in \Gamma$. The $p$-modulus of $\Gamma$ is defined by

$$
\bmod _{p}(\Gamma)=\inf \left\{\int_{X} \rho^{p}(x) d \mu: \rho: X \rightarrow[0, \infty] \text { is admissible for } \Gamma\right\} .
$$

We say that a condition holds for $p$-almost every path in $\Gamma$ if $\bmod _{p}(\hat{\Gamma})=0$, where $\hat{\Gamma} \subset \Gamma$ consists of those paths $\gamma \in \Gamma$ for which this condition fails. 
The $p$-modulus is an outer measure in the collection of all path families in $X$. For the basic properties of the $p$-modulus we refer the reader to [HeK2], [HeKST2].

Let $(X, d, \mu)$ be a metric measure space and $\left(Y, d_{Y}\right)$ be a metric space. Given an open set $U \subset X$ and a continuous mapping $f: U \rightarrow Y$, we say that a non-negative Borel-function $g$ is an upper gradient of $f$ in $U$ if, for each rectifiable path $\gamma:[0,1] \rightarrow U$, we have that

$$
d_{Y}(f(\gamma(1)), f(\gamma(0))) \leq \int_{\gamma} g d s .
$$

We recall here that each such $\gamma$ can be parametrized by a 1-Lipschitz map $\tilde{\gamma}:[0, L] \rightarrow U$. For $1 \leq p<\infty$, we call a non-negative Borel-function $g$ a $p$-weak upper gradient of $f$ in $U$ if the above inequality holds for $p$-almost every rectifiable path in $U$. It then follows from the properties of the $p$ modulus that (2) also holds for each subpath of $p$-almost every $\gamma$ in $U$. The existence of a $p$-integrable $p$-weak upper gradient always guarantees the existence of a $p$-integrable upper gradient, see [KosM]. Let $f: X \rightarrow Y$ be continuous. Then $f$ is in the Sobolev space $W_{l o c}^{1, p}(X ; Y)$ if, for each relatively compact open set $U \subset X, f$ has an upper gradient $g \in L^{p}(U)$ in $U$, and if there is $x_{0} \in U$ so that $u(x)=d_{Y}\left(f(x), f\left(x_{0}\right)\right) \in L^{p}(U)$. In what follows, we will typically have $\mu(U)<\infty$, and thus only a $p$-integrable $p$-weak upper gradient is asked for. Notice that for a proper $X$, each $f \in W_{l o c}^{1, p}(X ; Y)$ is absolutely continuous on $p$-almost every rectifiable path. For the purposes of this paper it suffices to consider continuous mappings $f$; for the definition and properties of general Sobolev classes we refer the reader to [HeKST2].

Because of the importance of $p$-weak upper gradients, we now give a sufficient condition for a path family to be of $p$-modulus zero.

Given a set $E \subset X$ and a path $\gamma: I \rightarrow X$, we let $\sharp(\gamma \cap E)$ denote the cardinality of $\gamma(I) \cap E$. We also write $\Gamma_{\text {rect }}$ for the collection of all rectifiable paths in $X$. We denote the $\lambda$-dimensional Hausdorff measure by $\mathcal{H}^{\lambda}$, and say that a set $E \subset X$ has $\sigma$-finite $\mathcal{H}^{\lambda}$-measure if it is contained in a countable union of sets with finite $\mathcal{H}^{\lambda}$-measure.

Proposition 3.1. Let $(X, d, \mu)$ be a proper, locally $Q$-regular metric space, $E \subset X$ and $1<p<Q$. If $E$ has $\sigma$-finite $\mathcal{H}^{Q-p}$-measure, then

$$
\bmod _{p}\left(\left\{\gamma \in \Gamma_{\text {rect }}: \gamma \cap E \text { is not countable }\right\}\right)=0 \text {. }
$$

We do not know if Proposition 3.1 could also hold for $p=1$, but the following version of it will be sufficient for our needs.

Proposition 3.2. Let $f: X \rightarrow Y$ be a homeomorphism between metric spaces, where $X$ is proper and locally $Q$-regular. Let $E \subset X$ have $\sigma$-finite 
$\mathcal{H}^{Q-1}$-measure. Then

$$
\bmod _{1}\left(\left\{\gamma \in \Gamma_{\text {rect }}: \mathcal{H}^{1}(f(\gamma \cap E))>0\right\}\right)=0 .
$$

For the proofs of these propositions we need a technical result that will also be applied later on.

Lemma 3.3. Let $(X, d, \mu)$ be a proper, locally $Q$-regular metric space. Further, let $B_{0}=B\left(x_{0}, r_{0}\right)$ and fix $1 \leq p<\infty$. There are constants $\delta$ and $C$ so that, given any collection $B_{1}, B_{2}, \cdots$ of balls in $B_{0}$ with radii at most $\delta$ and non-negative numbers $a_{i}$, we have the estimate

$$
\int_{B_{0}}\left(\sum_{i} a_{i} \chi_{6 B_{i}}(x)\right)^{p} d \mu \leq C \int_{B_{0}}\left(\sum_{i} a_{i} \chi_{B_{i}}(x)\right)^{p} d \mu .
$$

In fact, we may take $\delta=\frac{1}{6} \delta_{0}$, where $\delta_{0}$ is the constant for $B_{0}$ in the local $Q$-regularity condition.

For $p=1$ the claim immediately follows from the local $Q$-regularity and measure comparison. The general case is obtained using the $L^{p}-L^{p /(p-1)_{-}}$ duality and the boundedness of an appropriate restricted maximal function. In fact, already the doubling of the measure $\mu$ for scales up to $6 \delta$ would be sufficient; see [Bo] for a proof that generalizes to our setting.

A crucial step in proving Proposition 3.1 is the following result.

LEMma 3.4. Let $(X, d, \mu)$ be a proper, locally $Q$-regular metric space, $E \subset X$ bounded and $1<p<Q$. Denote by $\Gamma$ the collection of all rectifiable curves $\gamma$ in $X$ such that $\sharp(\gamma \cap E)=\infty$. If $\mathcal{H}^{Q-p}(E)<\infty$, then $\bmod _{p}(\Gamma)=0$.

Proof. Fix a ball $B_{0}$ so that $E \subset B_{0}$. Set for $k, l \in \mathbb{N}$

$$
\Gamma_{k, l}=\left\{\gamma \in \Gamma: \exists\left\{x_{1}, x_{2}, \ldots, x_{k}\right\} \in \gamma \cap E \text { s.t. } d\left(x_{i}, x_{j}\right)>\frac{1}{l} \text { when } i \neq j\right\} \text {. }
$$

Then $\Gamma=\cap_{k} \cup_{l} \Gamma_{k, l}$ and $\Gamma_{k, l} \subset \Gamma_{k, l+1}$. Thus

$$
\bmod _{p}\left(\cup_{l} \Gamma_{k, l}\right)=\lim _{l \rightarrow \infty} \bmod _{p}\left(\Gamma_{k, l}\right)
$$

(cf. [HeKST1]).

Fix $k$ and $l$ and let $\varepsilon>0$. Since $\mathcal{H}^{Q-p}(E)<\infty$, there is a cover of $E$ by balls $\left(B_{i}\right)_{i}$ such that $\operatorname{diam}\left(5 B_{i}\right)<1 / 2 l, E \subset \cup_{i} B_{i}$ and $\sum_{i} r_{i}^{Q-p} \leq$ $\mathcal{H}^{Q-p}(E)+\varepsilon$. We may assume that $B_{i} \subset B_{0}$ and that $\operatorname{diam}\left(5 B_{i}\right)<\delta$, where $\delta$ is the constant for $B_{0}$ in the local $Q$-regularity condition.

By Lemma 2.1, we find a subfamily of these balls (denoted the same way) that are pairwise disjoint and with $E \subset \cup_{i} 5 B_{i}$. Set

$$
\rho(x)=\frac{1}{k} \sum_{i} \frac{1}{r_{i}} \chi_{6 B_{i}}(x) .
$$


Now $\rho$ is admissible for $\Gamma_{k, l}$, since we have $\int_{\gamma} \chi_{6 B_{i}} d s \geq r_{i}$ for at least $k$ different indices. By Lemma 3.3 we have that

$$
\int_{X} \rho^{p}(x) d \mu \leq \frac{C}{k^{p}} \int_{X}\left(\sum_{i} \frac{1}{r_{i}} \chi_{B_{i}}(x)\right)^{p} d \mu .
$$

Here $C$ depends on $Q, p, B_{0}$. Since the balls $B_{i}$ are pairwise disjoint, by the local $Q$-regularity of $\mu$ we obtain

$$
\int_{X} \rho^{p}(x) d \mu \leq \frac{C}{k^{p}} \sum_{i} r_{i}^{Q-p} \leq \frac{C}{k^{p}}\left(\mathcal{H}^{Q-p}(E)+\varepsilon\right) .
$$

Our $\varepsilon$ was arbitrary, and thus

$$
\bmod _{p}\left(\cup_{l} \Gamma_{k, l}\right)=\lim _{l \rightarrow \infty} \bmod _{p}\left(\Gamma_{k, l}\right) \leq \frac{C}{k^{p}} \mathcal{H}^{Q-p}(E) .
$$

Since $\Gamma \subset \cup_{l} \Gamma_{k, l}$ for every $k$, the claim follows.

We continue with the case $p=1$. The difficulity in extending the above proof to this case lies in inequality (5). It only holds for $p>1$, the basic issue being reflexivity of $L^{p}$.

Lemma 3.5. Let $f: X \rightarrow Y$ be a homeomorphism between metric spaces, and assume that $X$ is proper and locally $Q$-regular. Let $E \subset X$ have finite $\mathcal{H}^{Q-1}$-measure. Fix $\varepsilon>0$. Then

$$
\bmod _{1}\left(\left\{\gamma \in \Gamma_{\text {rect }}: \mathcal{H}^{1}(f(\gamma \cap E))>\varepsilon\right\}\right)=0 .
$$

Proof. Denote our family of curves by $\Gamma_{\varepsilon}$. By subadditivity, we may without loss of generality assume that $E \subset B_{0}$ for some ball $B_{0}$. Let $k \geq 1$ be an integer. Because $\bar{B}_{0}$ is compact and $f$ is continuous, we find $\delta_{k}>0$ so that, given $x, x^{\prime} \in \bar{B}_{0}$ with $d_{X}\left(x, x^{\prime}\right)<\delta_{k}$, we have that

$$
d_{Y}\left(f(x), f\left(x^{\prime}\right)\right)<\frac{\varepsilon}{2^{k+3}} .
$$

As in the proof of the previous lemma, we find a sequence of balls $\left(B_{i}^{k}\right)_{i}$ so that $B_{i}^{k} \cap B_{j}^{k}=\emptyset$ when $i \neq j, \operatorname{diam}\left(B_{i}^{k}\right) \leq \frac{1}{6} \min \left\{\delta, \delta_{k}\right\}, E \subset \cup_{i} 5 B_{i}^{k}$ and so that

$$
\sum_{i}\left(\operatorname{diam}\left(5 B_{i}^{k}\right)\right)^{Q-1}<5^{Q-1} \mathcal{H}^{Q-1}(E)+\varepsilon .
$$

Consider the sequence $\left(\rho_{k}\right)_{k}$ of Borel-functions, defined by

$$
\rho_{k}(x)=\frac{1}{2^{k}} \sum_{i} \frac{1}{\operatorname{diam}\left(B_{i}^{k}\right)} \chi_{6 B_{i}^{k}}(x) .
$$

By (9) we see that

Set

$$
\int_{X} \rho_{k} d \mu \leq \frac{C}{2^{k}}\left(\mathcal{H}^{Q-1}(E)+\varepsilon\right) .
$$

$$
\Gamma_{(k)}=\left\{\gamma \in \Gamma_{\varepsilon}: \int_{\gamma} \rho_{k} d s \geq 1\right\} .
$$


By (10) and subadditivity we conclude that

$$
\bmod _{1}\left(\cup_{j \geq k} \Gamma_{(j)}\right) \leq \frac{C}{2^{k}}\left(\mathcal{H}^{Q-1}(E)+\varepsilon\right) .
$$

Let $\gamma \in \Gamma_{\varepsilon}$. Since $\mathcal{H}^{1}(f(\gamma \cap E))>\varepsilon$, for all sufficiently large integers $m$ there exist points $y_{1}, \ldots, y_{2^{m}} \in f(\gamma \cap E)$ so that

$$
d_{Y}\left(y_{i}, y_{j}\right)>\frac{\varepsilon}{2^{m+3}} \text {. }
$$

Notice that, by (8), there have to be at least $2^{m}$ balls $5 B_{i}^{m}$ in our sum which intersect $\gamma$. Thus,

$$
\int_{\gamma} \rho_{m} d s \geq 1
$$

It follows that $\Gamma_{\varepsilon} \subset \cup_{j \geq k} \Gamma_{(j)}$, for each $k$. From (11) we then conclude that $\bmod _{1}\left(\Gamma_{\varepsilon}\right)=0$.

Proof of Proposition 3.1. Suppose that $p>1$. We may write $E=\cup_{i} E_{i}$, where each $E_{i}$ is bounded with $\mathcal{H}^{Q-p}\left(E_{i}\right)<\infty$. Write

$$
\Gamma_{i}=\left\{\gamma \in \Gamma_{\text {rect }}: \gamma \cap E_{i} \text { is not countable }\right\} .
$$

By Lemma $3.4, \bmod _{p}\left(\Gamma_{i}\right)=0$, and the claim follows by subadditivity of the $p$-modulus.

Proof of Proposition 3.2. Lemma 3.5 shows that $\bmod _{p}\left(\Gamma_{1 / j}\right)=0$ for each $j \geq 1$. Here $\Gamma_{1 / j}$ consists of those rectifiable curves for which $\mathcal{H}^{1}(f(\gamma \cap E))>$ $1 / j$. The claim follows by subadditivity.

\section{Absolute Continuity on Almost All Paths}

We begin with the proof of Theorem 1.1.

Proof of Theorem 1.1. Let us denote the $Q$-regular Borel measures associated to $X$ and $Y$ by $\mu$ and $\nu$ respectively. Let $B_{0}=B\left(x_{0}, r_{0}\right)$ be a fixed ball. Without loss of generality assume that $H>1$. For each $k=1,2, \ldots$ write

$$
A_{k}=\left\{x \in B\left(x_{0}, r_{0}\right): H^{k}<h_{f}(x) \leq H^{k+1}\right\} .
$$

The set $A_{k}$ is a Borel set since $h_{f}$ is a Borel function. Moreover $\mu\left(\cup_{k} A_{k}\right)=0$. Fix $1<p<Q$, and let $0<\varepsilon<\varepsilon_{0}$, where $0<\varepsilon_{0}<\delta$ with $\delta$ from the local $Q$-regularity condition for $\bar{B}\left(x_{0}, r_{0}\right)$. Choose $\varepsilon_{0}$ so small that $f\left(B\left(x, \varepsilon_{0}\right)\right) \subset B\left(f(x), \delta^{\prime}\right)$ for each $x \in \bar{B}\left(x_{0}, r_{0}\right)$ for the constant $\delta^{\prime}$ from the local $Q$-regularity condition for $f\left(\bar{B}\left(x_{0}, r_{0}\right)\right)$.

Since $\mu\left(A_{k}\right)=0$, for every $k$ there exists an open set $U_{k}$ such that $A_{k} \subset U_{k}$ and

$$
\mu\left(U_{k}\right) \leq \frac{1}{H^{2 k p Q /(Q-p)}}\left(\frac{1}{2^{k} \nu\left(f\left(B\left(x_{0}, r_{0}+\varepsilon\right)\right)\right)^{p / Q}}\right)^{Q /(Q-p)},
$$


since we assumed that our measure is Borel regular. Notice here that $\nu\left(f\left(B\left(x_{0}, r_{0}+\varepsilon\right)\right)\right)$ is finite since $X$ is proper and $\nu$ is locally $Q$-regular.

For points $x \in B\left(x_{0}, r_{0}\right) \backslash\left(\cup_{k} A_{k} \cup E\right)$ pick a radius $0<r_{x}<\varepsilon$ such that $H_{f}\left(x, r_{x}\right) \leq 2 H$. For points $x \in A_{k}$ choose a radius $0<r_{x}<\varepsilon$ such that $H_{f}\left(x, r_{x}\right) \leq 2 H^{k+1}$ and $B\left(x, r_{x}\right) \subset U_{k}$. Now consider the family $\left\{B\left(x, r_{x}\right)\right\}$ of balls. By applying Lemma 2.2 for these balls we find a countable collection $\mathcal{B}=\left\{B\left(x_{i}, r_{i}\right)\right\}$ such that $B\left(x_{0}, r_{0}\right) \backslash E \subset \cup_{B \in \mathcal{B}} B$ and $\frac{1}{3} B, B \in \mathcal{B}$, are pairwise disjoint. Denote by $\mathcal{B}_{H}$ the subcollection of the balls $B\left(x, r_{x}\right) \in \mathcal{B}$ for which $x \in B\left(x_{0}, r_{0}\right) \backslash\left(\cup_{k} A_{k} \cup E\right)$ and by $\mathcal{B}_{k}$ those for which $x \in A_{k}$.

Relying on Lemma 2.3 we notice that

(i) $B\left(f\left(x_{i}\right), \frac{L_{f}\left(x_{i}, r_{i}\right)}{250 H^{2}}\right)$, where $B\left(x_{i}, r_{i}\right) \in \mathcal{B}_{H}$, are pairwise disjoint; and

(ii) $B\left(f\left(x_{i}\right), \frac{L_{f}\left(x_{i}, r_{i}\right)}{250 H^{2 k+2}}\right)$, where $B\left(x_{i}, r_{i}\right) \in \mathcal{B}_{k}$, are pairwise disjoint.

Set

$$
\rho_{\varepsilon}(x)=\sum_{i} \frac{L_{f}\left(x_{i}, r_{i}\right)}{r_{i}} \chi_{2 B_{i}}(x) .
$$

The function $\rho_{\varepsilon}$ is clearly Borel measurable. Let $\Gamma_{\varepsilon}$ denote all rectifiable paths $\gamma:[0,1] \rightarrow B\left(x_{0}, r_{0}\right)$ such that $\mathcal{H}^{1}(f(\gamma \cap E))=0$ and $\operatorname{diam}(\gamma)>\varepsilon$.

Let $\gamma$ be a member of $\Gamma_{\varepsilon}$. If $B_{i} \cap \gamma \neq \emptyset$, then $\mathcal{H}^{1}\left(\gamma \cap 2 B_{i}\right) \geq r_{i}$. Thus $\int_{\gamma} \rho_{\varepsilon} d s \geq \sum_{B_{i} \cap \gamma \neq \emptyset} L_{f}\left(x_{i}, r_{i}\right) \geq \frac{1}{2} \sum_{B_{i} \cap \gamma \neq \emptyset} \operatorname{diam}\left(f B_{i}\right) \geq \frac{1}{2} d_{Y}(f(\gamma(0)), f(\gamma(1)))$, where the last inequality comes from the fact that the sets $f\left(B_{i}\right)$ cover $f(\gamma)$ up to a set of zero $\mathcal{H}^{1}$-measure.

By Lemma 3.3 and the pairwise disjointness of the balls $\frac{1}{3} B_{i}$, we have the estimate

$$
\int_{X} \rho_{\varepsilon}^{p} d \mu \leq C \int_{B\left(x_{0}, r_{0}+2 \varepsilon\right)} \sum_{i} \frac{L_{f}\left(x_{i}, r_{i}\right)^{p}}{r_{i}^{p}} \chi_{\frac{1}{3} B_{i}} d \mu
$$

where $C=C\left(B_{0}, \mu, p\right)$.

Next we estimate this integral from above in two parts. First we consider the sum over $\mathcal{B}_{H}$-terms, which we denote by $S_{H}$. By Hölder's inequality we have

$$
\begin{aligned}
S_{H} & =C \int_{B\left(x_{0}, r_{0}+2 \varepsilon\right)} \sum_{B_{i} \in \mathcal{B}_{H}} \frac{L_{f}\left(x_{i}, r_{i}\right)^{p}}{r_{i}^{p}} \chi_{\frac{1}{3} B_{i}} d \mu \\
& \leq C \mu\left(B\left(x_{0}, r_{0}+2 \varepsilon\right)\right)^{\frac{Q-p}{Q}}\left(\int_{B\left(x_{0}, r_{0}+2 \varepsilon\right)}\left(\sum_{B_{i} \in \mathcal{B}_{H}} \frac{L_{f}\left(x_{i}, r_{i}\right)^{p}}{r_{i}^{p}} \chi_{\frac{1}{3} B_{i}}\right)^{\frac{Q}{p}} d \mu\right)^{\frac{p}{Q}} \\
& \leq C \mu\left(B\left(x_{0}, r_{0}+2 \varepsilon\right)\right)^{\frac{Q-p}{Q}}\left(\sum_{B_{i} \in \mathcal{B}_{H}} L_{f}\left(x_{i}, r_{i}\right)^{Q}\right)^{p / Q} .
\end{aligned}
$$


The last inequality comes from the pairwise disjointness of $\frac{1}{3} B_{i}$ 's and local $Q$-regularity of $\mu$. Since, by (i), the balls $B\left(f\left(x_{i}\right), L_{f}\left(x_{i}, r_{i}\right) / 250 H^{2}\right)$ with $B\left(x_{i}, r_{i}\right) \in \mathcal{B}_{H}$ are pairwise disjoint and $Y$ is locally $Q$-regular, we obtain the estimate

$$
S_{H} \leq C \mu\left(B\left(x_{0}, r_{0}+2 \varepsilon\right)\right)^{\frac{Q-p}{Q}} \nu\left(f\left(B\left(x_{0}, r_{0}+\varepsilon\right)\right)\right)^{\frac{p}{Q}}<\infty,
$$

where $C=C\left(B_{0}, \mu, f\left(B_{0}\right), \nu, p, H\right)$. The fact that the above term is finite comes from the properness of $X$ and local $Q$-regularity of both measures.

Next we need to estimate from above the sum over $\mathcal{B}_{k}$ 's. We denote the sum corresponding to $\mathcal{B}_{k}$ by $S_{k}$. Using the local $Q$-regularity and Hölder's inequality we obtain

$$
\begin{aligned}
S_{k} & \leq C \sum_{B_{i} \in \mathcal{B}_{k}} \frac{L_{f}\left(x_{i}, r_{i}\right)^{p}}{H^{2 k p+2 p}} H^{2 k p} r_{i}^{Q-p} \\
& \leq C\left(\sum_{B_{i} \in \mathcal{B}_{k}}\left(\frac{L_{f}\left(x_{i}, r_{i}\right)}{H^{2 k+2}}\right)^{Q}\right)^{\frac{p}{Q}}\left(\sum_{B_{i} \in \mathcal{B}_{k}} H^{2 k p Q /(Q-p)} \mu\left(\frac{1}{3} B_{i}\right)\right)^{\frac{Q-p}{Q}},
\end{aligned}
$$

where $C=C\left(B_{0}, \mu, p, H\right)$. Now, by (ii), the balls $B\left(f\left(x_{i}\right), L_{f}\left(x_{i}, r_{i}\right) / 250 H^{2 k+2}\right)$ with $B\left(x_{i}, r_{i}\right) \in \mathcal{B}_{k}$, are pairwise disjoint, and therefore the first term is no more than $C \nu\left(f\left(B\left(x_{0}, r_{0}+\varepsilon\right)\right)\right)^{p / Q}$. For the second term we use (12), so that

$$
S_{k} \leq C\left(\nu\left(f\left(B\left(x_{0}, r_{0}+\varepsilon\right)\right)\right)\right)^{\frac{p}{Q}} \frac{1}{2^{k} \nu\left(f\left(B\left(x_{0}, r_{0}+\varepsilon\right)\right)\right)^{p / Q}} \leq \frac{C}{2^{k}} .
$$

Thus

$$
\begin{aligned}
\int_{X} \rho_{\varepsilon}^{p} d \mu & \leq C\left(S_{H}+\sum_{k} S_{k}\right) \\
& \leq C \mu\left(B\left(x_{0}, r_{0}+2 \varepsilon\right)\right)^{\frac{Q-p}{Q}} \nu\left(f\left(B\left(x_{0}, r_{0}+\varepsilon\right)\right)\right)^{\frac{p}{Q}}+C .
\end{aligned}
$$

So, for all paths $\gamma \in \Gamma_{\varepsilon}$, we have the estimate

$$
d_{Y}(f(\gamma(0)), f(\gamma(1))) \leq \int_{\gamma} 2 \rho_{\varepsilon} d s
$$

with $\int\left(2 \rho_{\varepsilon}\right)^{p} d \mu \leq M<\infty$ when $0<\varepsilon<\varepsilon_{0}$. The weak compactness of $L^{p}$ guarantees that there is $\rho \in L^{p}$ and a sequence of $\varepsilon_{i}$ 's that decreases to zero such that $\rho$ is a $L^{p}$-weak limit of $2 \rho_{\varepsilon_{i}}=: \rho_{i}$. Here we needed the fact that $p>1$. Notice that

$$
d_{Y}(f(\gamma(0)), f(\gamma(1))) \leq \int_{\gamma} \rho_{i} d s
$$

for each $i \geq j$ when $\gamma \in \Gamma_{j}:=\Gamma_{\varepsilon_{j}}$. By Mazur's lemma (cf. [Y, Ch.V.1, Th. 2]), we find functions $\hat{\rho}_{i}$, each a convex combination of $\rho_{i}, \rho_{i+1}, \ldots$, so 
that the sequence $\left\{\hat{\rho}_{i}\right\}$ converges to $\rho$ in $L^{p}$. Now (13) also holds with $\rho_{i}$ replaced with $\hat{\rho}_{i}$ for each $i \geq j$. By Fuglede's lemma (cf. [HeKST1]), (13) holds for $\rho$ for $p$-almost every $\gamma \in \cup_{j} \Gamma_{j}$. Thus, using Proposition 3.2, we notice that (13) holds for 1-almost every rectifiable curve in $B\left(x_{0}, r_{0}\right)$; recall that we excluded the curves for which $\mathcal{H}^{1}(f(\gamma \cap E))>0$. Thus $f$ has a 1-weak upper gradient in $L^{1}\left(B\left(x_{0}, r_{0}\right)\right)$, and consequently an upper gradient in $L^{1}\left(B\left(x_{0}, r_{0}\right)\right)$. In conclusion, $f \in W^{1,1}\left(B\left(x_{0}, r_{0}\right) ; Y\right)$, and the theorem is proven.

REMARK 4.1. The above proof immediately gives the following variations on Theorem 1.1. First of all, if we consider a smaller exceptional set $E$ which is of $\sigma$-finite $\mathcal{H}^{Q-p}$-measure, $1<p<Q$, we can conclude (using Proposition 3.1 instead of Proposition 3.2) that $f \in W_{l o c}^{1, p}(X ; Y)$. In the borderline case $p=Q$, one can still conclude that $f \in W_{l o c}^{1, Q}(X ; Y)$ but one can only allow for a countable exceptional set $E$ and the bound $h_{f}(x) \leq H$ needs to be assumed for each $x \in X \backslash E$. For a further improvement on this see Theorem 5.1 and Remarks 5.3 below.

The proof of Theorem 1.1 is not constructive in the sense that no explicit upper gradient is given. Classically, one can take the maximal streching

$$
L_{f}(x)=\limsup _{r \rightarrow 0} \frac{L_{f}(x, r)}{r}
$$

as an upper gradient. In our situation it turns out to be better to consider the volume derivative

$$
\mu_{f}(x)=\lim _{r \rightarrow 0} \frac{\nu(f(B(x, r)))}{\mu(B(x, r))} .
$$

Recall that, by the Lebesgue-Radon-Nikodym theorem $[\mathrm{F}]$, this limit exists almost everywhere and we have the estimate

$$
\int_{A} \mu_{f}(x) d \mu \leq \nu(f(A))
$$

for each measurable set $A \subset X$. Consequently,

$$
\int_{A} u(f(x)) \mu_{f}(x) d \mu \leq \int_{f(A)} u(y) d \nu
$$

when $u \geq 0$ is continuous.

For technical reasons, we assume from now on a global condition on the measures $\mu$ and $\nu$. We say that a measure $\mu$ on a metric space $X$ is $Q$-regular if it is locally $Q$-regular with a universal constant $C_{\mu}$ and with $\delta=\operatorname{diam}(X)$, i.e.

$$
\frac{1}{C_{\mu}} r^{Q} \leq \mu(B(x, r)) \leq C_{\mu} r^{Q}
$$

holds for each $x$ and all $0<r<\operatorname{diam}(X)$. 
Proposition 4.2. Let $\left(X, d_{X}, \mu\right)$ and $\left(Y, d_{Y}, \nu\right)$ be $Q$-regular metric spaces, $Q>1$. Assume also that $X$ is proper. Let $f: X \rightarrow Y$ be a homeomorphism such that $h_{f}(x) \leq H<\infty$ for $\mu$-almost every $x \in X$. Define

$$
g_{f}(x)= \begin{cases}H \limsup _{r \rightarrow 0}\left(\frac{\nu(f(B(x, r)))}{\mu(B(x, r))}\right)^{1 / Q} & \text { when } h_{f}(x) \leq H \\ \infty & \text { when } h_{f}(x)>H\end{cases}
$$

If $f$ is absolutely continuous on $\gamma:[0, L] \rightarrow X$, which is 1-Lipschitz, then

$$
d_{Y}(f(\gamma(0)), f(\gamma(L))) \leq C \int_{\gamma} g_{f} d s
$$

where $C$ depends only on the $Q$-regularity constants of $\mu, \nu$.

Proof. Notice that $g_{f}$ is Borel measurable since $h_{f}$ is Borel measurable.

Let $y \in \gamma(] 0, L[)$. Then there are arbitrarily small $r_{y}>0$ so that

$$
f\left(B\left(y, r_{y}\right)\right) \subset B\left(f(y), L_{f}\left(y, r_{y}\right)\right) \subset B\left(f(y), 2 h_{f}(y) l_{f}\left(y, r_{y}\right)\right) .
$$

We might have here that $h_{f}(y)=\infty$, but that will not harm us. Let $x \in] 0, L[$ and write $y=\gamma(x)$. Because $\gamma$ is 1-Lipschitz we conclude that $\operatorname{diam}\left(f\left(\gamma(] x-r_{y}, x+r_{y}[)\right)\right) \leq 4 h_{f}(y) l_{f}\left(y, r_{y}\right)$

$$
\leq C h_{f}(y) \nu\left(f\left(B\left(y, r_{y}\right)\right)^{1 / Q}\right.
$$

where we used the $Q$-regularity of $\nu$.

Fix $\varepsilon>0$. For $i \in \mathbb{Z}$, let

$$
E_{i}=\{x \in] 0, L\left[: 2^{i-1}<g_{f}(\gamma(x)) \leq 2^{i}\right\} .
$$

Let $x \in E_{i}$. Using (16), the definition of $g_{f}$ and the $Q$-regularity of $\mu$, we may pick an arbitrarily small $r_{y}>0$ such that

$$
\operatorname{diam}\left(f\left(\gamma(] x-r_{y}, x+r_{y}[)\right) \leq C 2^{i} r_{y} .\right.
$$

Covering $E_{i}$ appropriately, we obtain intervals $I_{1}^{(i)}, I_{2}^{(i)}, \ldots$ so that $E_{i} \subset \cup_{j} I_{j}^{(i)}$ and

$$
\sum_{j} \operatorname{diam}\left(f\left(\gamma\left(I_{j}^{i}\right)\right)\right) \leq C \int_{\gamma \mid E_{i}} g_{f} d s+\frac{\varepsilon}{2^{|i|+2}} .
$$

Letting $i$ run through $\mathbb{Z}$, we end up with a collection $J_{1}, J_{2}, \ldots$ of intervals such that $\cup_{i} E_{i} \subset \cup_{j} J_{j}$ and

$$
\sum_{j} \operatorname{diam}\left(f\left(\gamma\left(J_{j}\right)\right)\right) \leq C \int_{\gamma} g_{f} d s+\varepsilon
$$

In order to obtain (15) we still need to consider the remaining parts of $] 0, L[$ where $g_{f}$ is either 0 or $\infty$.

Write $E^{0}=\{x \in] 0, L\left[: g_{f}(\gamma(x))=0\right\}$, and let $x \in E^{0}$. Then (16) implies that

$$
\liminf _{r \rightarrow 0} \frac{\operatorname{diam}(f(\gamma(] x-r, x+r[)))}{r}=0 .
$$


It follows that

$$
\mathcal{H}^{1}\left(f\left(\gamma\left(E^{0}\right)\right)\right)=0 .
$$

Write $E^{\infty}=\{x \in] 0, L\left[: g_{f}(\gamma(x))=\infty\right\}$. If $\mathcal{H}^{1}\left(E^{\infty}\right)>0$, then (15) is trivially true. Otherwise, $\mathcal{H}^{1}\left(E^{\infty}\right)=0$, and the absolute continuity of $f$ on $\gamma$ shows that

$$
\mathcal{H}^{1}\left(f\left(\gamma\left(E^{\infty}\right)\right)\right)=0 .
$$

The claim follows by combining (17), (18) and (19) and letting $\varepsilon \rightarrow 0$.

Remark 4.3. Combining Proposition 4.2 and Remark 4.1 it is easy to check that the uniform boundedness of $h_{f}$ implies the so-called geometric definition (see [G2]) for proper $Q$-regular spaces. Notice also that, in the Euclidean setting, it follows that $f$ is quasiconformal if and only if there is a constant $K$ so that $K \mu_{f}^{1 / Q}$ is a $Q$-weak upper gradient of $f$.

\section{$5 \quad W_{l o c}^{1, Q}$ and Quasisymmetry}

Let us recall that $(X, d, \mu)$ supports a $p$-Poincaré inequality, $1 \leq p<\infty$ if there are two constants $C$ and $\tau$ such that

$$
\frac{1}{\mu(B(x, r))} \int_{B(x, r)}\left|u-u_{B(x, r)}\right| d \mu \leq C r\left(\frac{1}{\mu(B(x, \tau r))} \int_{B(x, \tau r)} g^{p} d \mu\right)^{1 / p}
$$

whenever $B(x, r)$ is a ball, $u$ is continuous and $g$ is an upper gradient of $u$. Here $u_{B(x, r)}$ is the average of $u$ over $B(x, r)$. It then follows that this also holds when $g$ is a $p$-weak upper gradient, see $[\operatorname{KosM}]$. For more on Poincaré inequalities see [HeK2], [HaK], [KeZ].

We begin by improving on the regularity of $f$ in Theorem 1.1.

Theorem 5.1. Let $\left(X, d_{X}, \mu\right)$ and $\left(Y, d_{Y}, \nu\right)$ be $Q$-regular metric spaces, $Q>1$, and suppose that $E \subset X$ has $\sigma$-finite $\mathcal{H}^{Q-1}$-measure. Assume also that $X$ is proper and supports a 1-Poincaré inequality. If $f: X \rightarrow Y$ is a homeomorphism such that $h_{f}(x)<\infty$ for every $x \in X \backslash E$ and $h_{f}(x) \leq$ $H<\infty$ for $\mu$-almost every $x \in X$, then $f \in W_{l o c}^{1, Q}(X ; Y)$.

Proof. From Theorem 1.1 we know that $f \in W_{l o c}^{1,1}(X ; Y)$, and from Proposition 4.2 that $g_{f} \in L_{l o c}^{Q}(X)$ is a 1 -weak upper gradient. Because $X$ supports a 1-Poincaré inequality, it follows that $f \in W_{l o c}^{1, Q}(X ; Y)$; see [KosM, Prop. 4.4] and [HeKST1, Th. 6.11].

We continue with a quasisymmetry result. This result could be proven by combining the ideas from the proof of Theorem 1.1 with the techniques in $[$ HeK1,2]. Instead of that we sketch a direct proof along classical lines using Theorem 1.1. 
Theorem 5.2. Let $\left(X, d_{X}, \mu\right)$ and $\left(Y, d_{Y}, \nu\right)$ be both proper, $Q$-regular metric spaces, $Q>1$, and $E \subset X$ such that it has $\sigma$-finite $\mathcal{H}^{Q-1}$-measure. Assume also that $X$ supports a 1-Poincaré inequality and that $Y$ is linearly locally connected. If $f: X \rightarrow Y$ is a homeomorphism such that $h_{f}(x)<\infty$ for every $x \in X \backslash E$ and $h_{f}(x) \leq H<\infty$ for almost every $x \in X$, then $f$ is quasisymmetric, $f \in W_{l o c}^{1, Q}(X ; Y)$, and $L_{f}(x)^{Q} \leq K \mu_{f}(x)$ almost everywhere for some constant $K$.

Proof. Fix $B\left(x_{0}, r_{0}\right)$, and suppose that $L:=L_{f}\left(x_{0}, r_{0}\right)$ is much larger than $l:=l_{f}\left(x_{0}, r_{0}\right)$.

From Theorem 1.1 and Proposition 4.2 we know that $f$ is absolutely continuous on 1-almost every path and that we may take $g_{f}$ as defined in Proposition 4.2 as an upper gradient.

Suppose that $E$ and $F$ are continua so that $y_{0} \in f(E) \subset B\left(y_{0}, C l\right)$ and $f(F) \subset Y \backslash \bar{B}\left(y_{0}, L / C\right)$ for some $y_{0} \in Y$. We define a function $u: X \rightarrow$ $[0, \infty[$ by setting

$$
u(x)=\frac{\log \left(L / C d_{Y}\left(f(x), y_{0}\right)\right)}{\log \left(L / C^{2} l\right)}
$$

in $A:=f^{-1}\left(\bar{B}\left(y_{0}, L\right) \backslash B\left(y_{0}, l\right)\right)$ and by extending $u$ to all of $X$ by setting $u=0$ in $X \backslash f^{-1}\left(\bar{B}\left(y_{0}, L / C\right)\right)$ and $u=1$ in $f^{-1}\left(\bar{B}\left(y_{0}, C l\right)\right)$. It easily follows that $g(x)=\log \left(L / C^{2} l\right)^{-1} d_{Y}\left(f(x), y_{0}\right)^{-1} g_{f}(x) \chi_{A}(x)$ is a 1-weak upper gradient of $u$.

$$
\begin{aligned}
\int_{X}^{\mathrm{Now}} g^{Q} d \mu & \leq C H^{Q} \log (L / l)^{-Q} \int_{A} d\left(f(x), y_{0}\right)^{-Q} \mu_{f}(x) d \mu(x) \\
& \leq C H^{Q} \log \left(L / C^{2} l\right)^{-Q} \int_{\bar{B}\left(y_{0}, L / C\right) \backslash B\left(y_{0}, C l\right)} d\left(y, y_{0}\right)^{-Q} d \nu(y) \\
& \leq C \log \left(L / C^{2} l\right)^{1-Q}
\end{aligned}
$$

by (14) and the $Q$-regularity of $Y$.

On the other hand, the Poincaré inequality and $Q$-regularity assumptions on $Y$ allow us to choose the continua $E, F$ as above so that $\operatorname{diam}(E), \operatorname{diam}(F) \geq r_{0}, d(E, F) \leq 2 r_{0}$, where $C$ is a constant only depending on the $Q$-regularity constant, $Q$, and the constants in the linear local connectivity condition. For all this see [HeK2, §4] and [HaK, §4.4 and $\S 4.5]$. Because $g$ is a 1-weak upper gradient of $u$, we may apply the 1-Poincaré inequality to the pair $u, g$ and Theorem 5.9 in [HeK2] shows that

A bound on $L / l$ follows, as desired.

$$
\int_{X} g^{Q} d \mu \geq C>0 \text {. }
$$


Next, from Theorem 5.1 we know that $f \in W_{l o c}^{1, Q}(X ; Y)$.

The final conclusion immediately follows from the uniform boundedness of $H_{f}(x, r)$.

Remarks 5.3. 1) If, instead of a 1-Poincaré inequality on $X$, we assume a $p$-Poincaré inequality, $1<p<Q$, then the conclusions of Theorem 5.1,5.2 still hold provided we require that the exceptional set $E$ have $\sigma$-finite $\mathcal{H}^{Q-p_{-}}$ measure. Notice that a $Q$-Poincaré inequality for a space as in the above theorem always implies a $p$-Poincaré inequality for some $p<Q$ by a very recent result in $[\mathrm{KeZ}]$.

2) There is a local version of Theorem 5.2, whose formulation and proof we leave to the interested reader.

Proof of Theorem 1.2. A Carnot group of homogeneous dimension $Q>1$ is proper, $Q$-regular, and supports a 1-Poincaré inequality. Thus the claim follows from Theorem 5.2, except for the inequality for the horizontal differential. This inequality follows from the corresponding inequality in Theorem 5.2 and quasisymmetry ([KoR], $[\mathrm{P}],[\mathrm{VG}])$.

\section{References}

[A] L. Ahlfors, On quasiconformal mappings, J. Analyse Math. 3 (1954), $1-58$.

[BC] Z.M. Balogh, M. Csörnyei, Scaled oscillation and regularity, Proc. Amer. Math. Soc. 134:9 (2006), 2667-2675.

[BK] Z.M. Balogh, P. Koskela, Quasiconformality, quasisymmetry, and removability in Loewner spaces, with an appendix by J. VÄIsÄLё, Duke Math. J. 101 (2000), 554-577.

[BRS] Z.M. Balogh, M. Rickly, F. Serra Cassano, Comparison of Hausdorff measures with respect to the Euclidean and the Heisenberg metric, Publ. Mat. 47 (2003), 237-259.

[BT] Z.M. BALOGH, J.T. Tyson, Hausdorff dimension of self-similar and selfaffine fractals in the Heisenberg group, Proc. London Math. Soc. 91 (2005), $153-183$.

[Bo] B. Bojarski, Remarks on Sobolev imbedding inequalities, Proc. of the Conference on Complex Analysis, Joensuu 1987, Springer Lecture Notes in Math. 1351 (1988), 52-68.

[BouP] M. Bourdon, H. PAJot, Poincaré inequalities and quasiconformal structure on the boundary of some hyperbolic buildings, Proc. Amer. Math. Soc. 127 (1999), 2315-2324.

[F] H. Federer, Geometric Measure Theory, Grundlehren Math. Wiss. 153, Springer-Verlag, New York, 1969. 
[G1] F.W. Gehring, The definitions and exceptional sets for quasiconformal mappings, Ann. Acad. Sci. Fenn. Ser. AI Math. 281 (1960), 1-28.

[G2] F.W. GeHring, Rings and quasiconformal mappings in space, Trans. Amer. Math. Soc. 103 (1962), 353-393.

[GrS] J. GraczyK, S. SmiRnov, Non-uniform hyperbolicity in complex dynamics I,II, preprint.

[H] P. HaÏssinsky, Rigidity and expansion for rational maps, J. London Math. Soc. (2) 63 (2001), 128-140.

[HaK] P. HajŁasz, P. Koskela, Sobolev met Poincaré, Mem. Amer. Math. Soc. 145 (2000), 1-101.

[HeK1] J. Heinonen, P. Koskela, Definitions of quasiconformality, Invent. Math. 120 (1995), 61-79.

[HeK2] J. Heinonen, P. Koskela, Quasiconformal maps in metric spaces with controlled geometry, Acta Math. 181 (1998), 1-61.

[HeKST1] J. Heinonen, P. Koskela, N. Shanmugalingam, J.T. Tyson, Sobolev classes of Banach space-valued functions and quasiconformal mapings, J. Anal. Math. 85 (2001), 87-139.

[HeKST2] J. Heinonen, P. Koskela, N. Shanmugalingam, J.T. Tyson, Metric Sobolev spaces: An approach based on upper gradients, in prepartion.

[KK1] S. Kallunki, P. Koskela, Exceptional sets for the definition of quasiconformality, Amer. J. Math. 122 (2000), 735-743.

[KK2] S. Kallunki, P. Koskela, Metric definition of $\mu$-homeomorphisms, Michigan Math. J. 51 (2003), 141-151.

[KeZ] S. Keith, X. Zhong, The Poincaré inequality is an open ended condition, Annals of Math, to appear.

[KiS] B. Kirchheim, F. Serra Cassano, Rectifiability and parametrization of intrinsic regular surfaces in the Heisenberg group, Ann. Sc. Norm. Super. Pisa Cl. Sci. (5) 3 (2004), 871-896.

[KoR] A. Korányi, H.M. Reimann, Foundations for the theory of quasiconformal mappings on the Heisenberg group, Adv. Math. 111 (1995), 1-87.

[KosM] P. Koskela, P. MacManus, Quasiconformal mappings and Sobolev spaces, Studia Math. 131 (1998), 1-17.

[MM] G.A. Margulis, G.D. Mostow, The differential of a quasi-conformal mapping of a Carnot-Carathéodory space, GAFA, Geom. funct. anal. 5 (1995), 402-433.

[Ma] P. Mattila, Geometry of Sets and Measures in Euclidean Spaces, v. 44, Cambridge Studies in Advanced Mathematics, Cambridge University Press, 1995.

[Me] D. Menchoff, Sur une généralisation d'un théorème de M.H. Bohr, Mat. Sbornik 44 (1937), 339-354. 
[Mo1] G.D. Mostow, Quasiconformal mappings in $n$-space and the rigidity of hyperbolic space forms, Inst. Hautes Études Sci. Publ. Math. 34 (1968), 53-104.

[Mo2] G.D. Mostow, Strong Rigidity of Locally Symmetric Spaces, Annals of Mathematics Studies 78, Princeton University Press, Princeton, N.J., 1973.

[Mo3] G.D. Mostow, A remark on quasiconformal mappings on Carnot groups, Michigan Math. J. 41 (1994), 31-37.

[P] P. PAnsu, Métriques de Carnot-Carathéodory et quasiisométries des espaces symétriques de rang un, Ann. of Math. 129 (1989), 1-60.

[PrR] F. PRzytycki, S. Rohde, Rigidity of holomorphic Collet-Eckmann repellers, Ark. Mat. 37 (1999), 357-371.

[R] S. Rigot, Counter example to the Besicovitch covering property for some Carnot groups equipped with their Carnot-Carathéodory metric, Math. Z. 248 (2004), 827-848.

[VG] S.K. Vodopyanov, A.V. Greshnov, Analytic properties of quasiconformal mappings on Carnot groups, Siberian Math. J. 36 (1995), 1142-1151.

[Y] K. YosidA, Functional Analysis, Springer-Verlag, 1978.

Z.M. BALOGH, Universität Bern, Mathematisches Institut, Sidlerstrasse 5, CH3012 Bern, Switzerland

zoltan.balogh@math.unibe.ch

P. Koskela, Department of Mathematics and Statistics, P.O. Box 35, University of Jyväskylä, FIN-40014 Finland

pkoskela@maths.jyu.fi

S. Rogovin, Department of Mathematics and Statistics, P.O. Box 35, University of Jyväskylä, FIN-40014 Finland

sakallun@maths.jyu.fi

Received: August 2005

Accepted: January 2006 